\title{
Microstructural Stability of Duplex Stainless Steel Weldments
}

\author{
Janos Dobranszky ${ }^{1, a}$ and Janos Ginsztler ${ }^{1,2, b}$ \\ ${ }^{1}$ Research Group for Metals Technology of the Hungarian Academy of Sciences \\ Goldmann ter 3, Budapest 1118, Hungary \\ ${ }^{2}$ Budapest University of Technology and Economics, \\ Department of Materials Science and Engineering \\ Goldmann ter 3, Budapest 1118, Hungary \\ adobi@eik.bme.hu, bginsztler@mti.bme.hu
}

Keywords: ATIG welding, laser beam welding, duplex stainless steel, thermoelectric power measurement

\begin{abstract}
Welding experiences are demonstrated, which were developed on 2205 type duplex and 2509 type superduplex stainless steels (DSS and SDSS). The welded joints were produced by activated TIG welding and laser beam welding. For the ATIG process, which is a special kind of the TIG-welding, silica powder was used as activating flux. Microstructural characterizations were applied for the measurement of ferrite/austenite phase ratio in the weldments. The welded joints were thermally aged at temperature of $400{ }^{\circ} \mathrm{C}$. The phase transformations produced during thermal ageing were investigated by optical and scanning electron microscopy and thermoelectric power measurement. Investigations show that the thermal ageing induced phase transformations are similar at ATIG-welded weldment and DSS base material, but the incubation time is significantly shorter in the weldment than in the base material when laser welding is applied.
\end{abstract}

\section{Introduction}

Tungsten inert gas welding is one of the cleanest and most important welding procedures for welding of duplex stainless steels, but its productivity is not too significant. In order to improve the productivity, several developments and experimental procedures based on new suggestions were born. Developments may happen in two main ways. Automatic addition of consumables into the joint is one of them and improving the efficiency of the welding arc (resulting higher penetration) is an other way. Using of the activating fluxes for TIG welding also belongs to this second way of the developments. Laser beam welding has more and more significance, particularly after when the laserhybrid welding is presented.

When activating fluxes are used at Activated TIG (ATIG) welding, there are two significant properties. First, none of welding consumable is needed for ATIG welding. The second difference is the usage of activating fluxes. A few theories explain the mechanism of the activating fluxes: $\mathrm{Si}_{\text {- }}$ monik says that oxide and fluoride molecules are present in the activating fluxes, which have affinity to chain the free electrons at the edge of the plasma of the arc. Savitskij and Leskov say that the activating fluxes decrease the surface tension of the weld pool, which makes the arc pressure able to cause a deeper invading in the pool. Lowke, Tanaka and Ushio explain that the deep penetration can be thanked to the higher electric insulation of the activating fluxes [1]. Heiple and Roper explain the high penetration with the inverse Marangoni effect $[2,3]$.

As many phase transformations can happen during welding of these nitrogen containing high alloy steels, it is important to clarify the phase construction stability of weldments at the highest service temperature of DSS and SDSS [4,5]. There are intermetallic phases and nitrides, which's formation influence toughness and corrosion resistance of weldments [6-9]. Thermoelectric power (TEP) measurements can be applied for continuing of phase decompositions and other transformations in DSS and SDSS at high temperature [10-14]. The aim of this work is to investigate the sensitivity of TEP measurement for weldments, which were aged at moderate temperatures. 


\section{Experiments}

2205 type, $3 \mathrm{~mm}$ thick DSS plates (the chemical composition can be seen in the Table 1) were welded by ATIG welding at which the activating flux was silica powder. Butt-welded joints of 200 mm length were produced without joint gap between the plates. Pure Ar as shielding and $95 \% \mathrm{Ar}+$ $5 \% \mathrm{~N}_{2}$ mixture as backing gas were applied. Welding current and speed was $90 \mathrm{~A}$ and $21 \mathrm{~cm} / \mathrm{min}$. From the weldments a $100 \times 2 \times 3 \mathrm{~mm}$ measures sample was machined for the further investigations, e.g. TEP measurement and microscopy.

Laser-butt welding was carried out on 2509 type SDSS which's cross section was $0,5 \times 2 \mathrm{~mm}$. This means that the examined sample of $100 \mathrm{~mm}$ length contains a welded joint at its center. A TRUMPF PowerWeld laser welding system was used for the welding experiments. Welding parameters were: $0.7 \mathrm{~mm}$ spot size, $3.8 \mathrm{~ms}$ pulse duration, $11 \mathrm{~Hz}$ frequency, $1.4 \mathrm{~J}$ pulse energy and 2.4 $\mathrm{kW}$ power and 3 shot.

Fig. 1 and Fig. 2 show the microstructure of base metals and macrograph of weldments. Welded 2509 SDSS samples and 2205 DSS weldment were treated by thermal ageing at $400{ }^{\circ} \mathrm{C}$. Thermoelectric power measurements, optical microscopy and SEM-EDS analysis were carried out on the thermally aged samples.

\begin{tabular}{|c|c|c|c|c|c|c|c|c|c|c|c|c|}
\hline & $\mathbf{C}$ & $\mathbf{S i}$ & $\mathbf{M n}$ & $\mathbf{P}$ & $\mathbf{S}$ & $\mathbf{C r}$ & $\mathbf{N i}$ & $\mathbf{M o}$ & $\mathbf{N b}$ & $\mathbf{C u}$ & $\mathbf{C o}$ & $\mathbf{N}$ \\
\hline 2205 type DSS & 0.017 & 0.40 & 1.40 & 0.019 & 0.001 & 22.41 & 5.33 & 3.19 & 0.003 & 0.11 & 0.08 & 0.181 \\
\hline 2509 type SDSS & 0.002 & 0.44 & 0,37 & 0.018 & 0.001 & 25.24 & 9.93 & 3.99 & - & 0.03 & - & 0.27 \\
\hline
\end{tabular}

Table 1. Chemical composition of the investigated steels
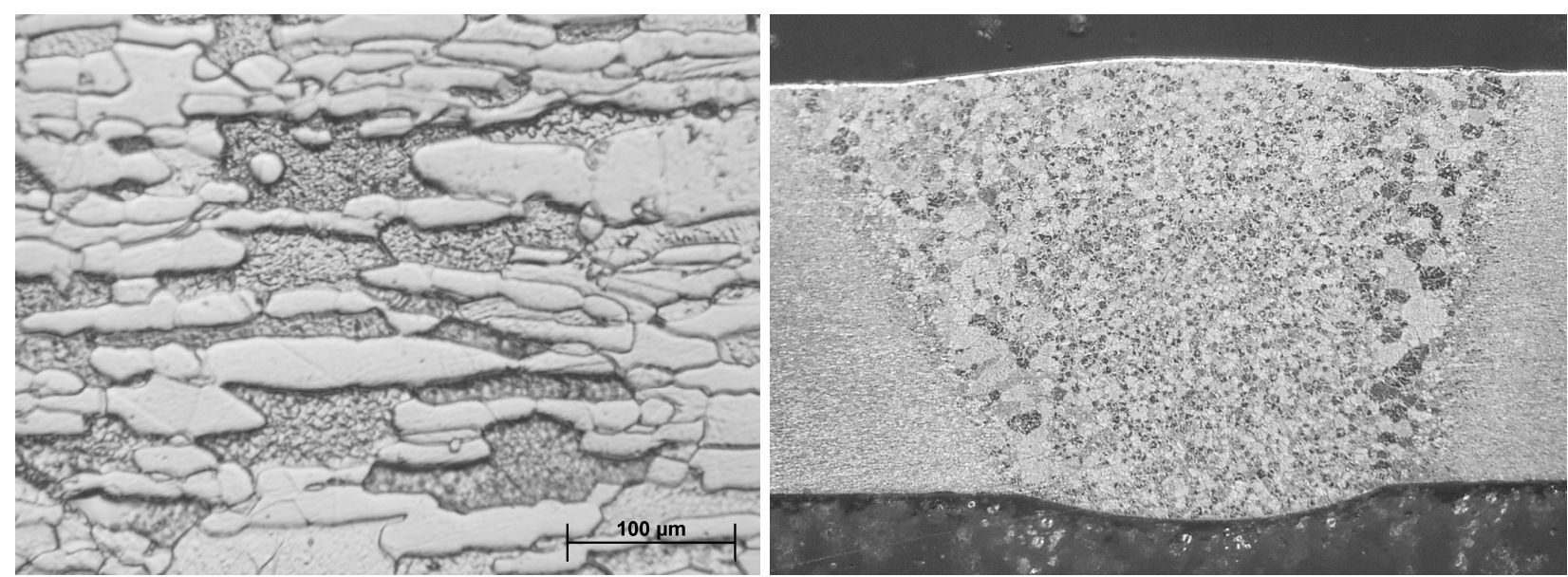

Fig. 1. Microstructure of 2205 type DSS and the cross section of ATIG-welded joint.
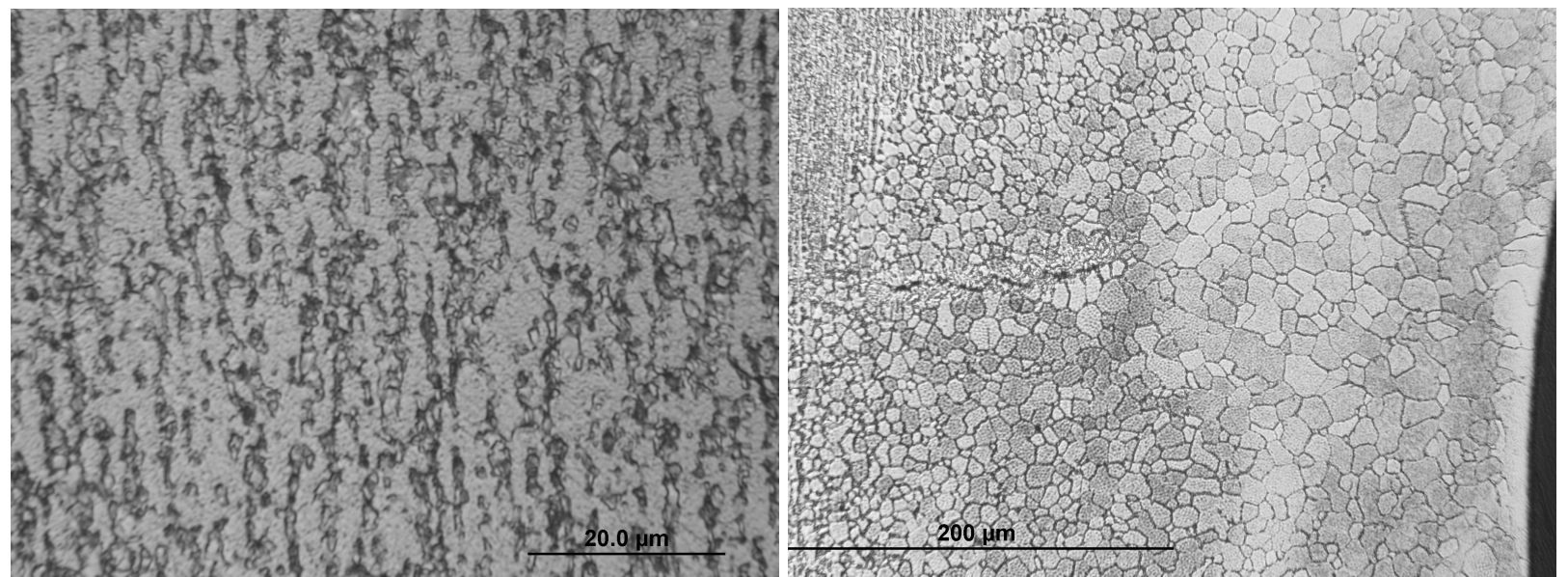

Fig. 2. Microstructure of 2509 type SDSS and the cross section of laser-butt welded joint. 


\section{Results and discussion}

Optical microscopy and SEM-EDS analysis of ATIG weldments and laser butt-welded samples in as-welded and thermally aged state (3300 minutes) do not show any characteristic microstructural changes. However, the analysis of thermoelectric power values make possible to detect characteristic points, which can be the incubation time and the finishing point of the main phase transformation (formatin of the $\alpha^{\prime}$ phase) at applied ageing temperature $\left(400^{\circ} \mathrm{C}\right.$ ).

It was examined how the TEP changes in function of ageing time and the derivative curves of TEP changing curves were also calculated. Where the first minimum of a derivative curve is found, there is the start point of the (TEP-sensitive) main phase transformation, and the next maximum signifies the end of this transformation. It may touch in that not only one phase transformation and other microstructural change can happen in the same time; TEM analysis will be carried out to clarify its precise character. The gradient of TEP changing curve only slowly approaches to zero at duplex steel but much more quickly at superduplex weldment.

In the case of 2205 DSS (see Fig. 3) the incubation time for base material and weldment is practically the same: 23-24 minutes, consequently it can be stated, that the ATIG-weldment has the same phase transformation comportment as the cold rolled base material. But in the laser buttwelded 2509 SDSS samples (see Fig. 4) the phase transformation was started much more quickly, 12,2 minutes later in the welded joints, than in the base material (22,3 minutes).

It is clearly seen that the microstructure of two different weld metal differs significantly; all the same TEP changing curves are very similar, which means that the transformations have the same character.
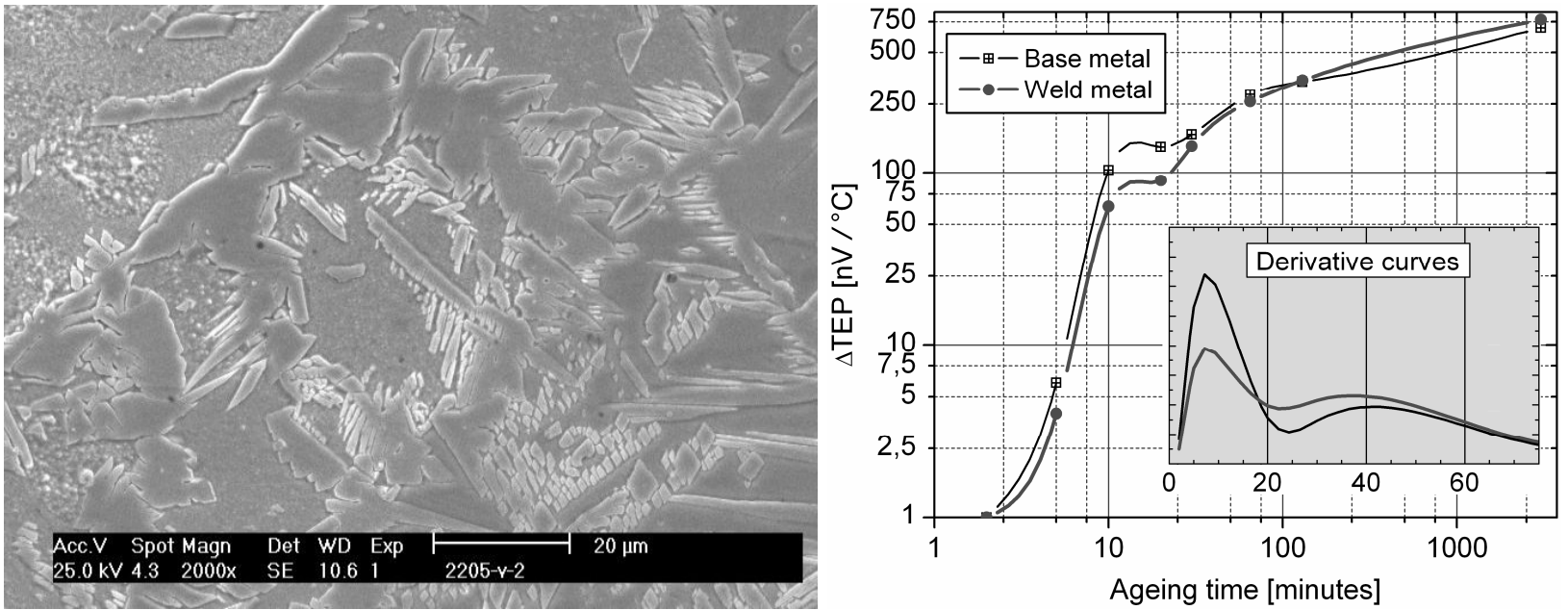

Fig. 3. Microstructure of ATIG weldment and the curves of TEP changing in function of ageing time
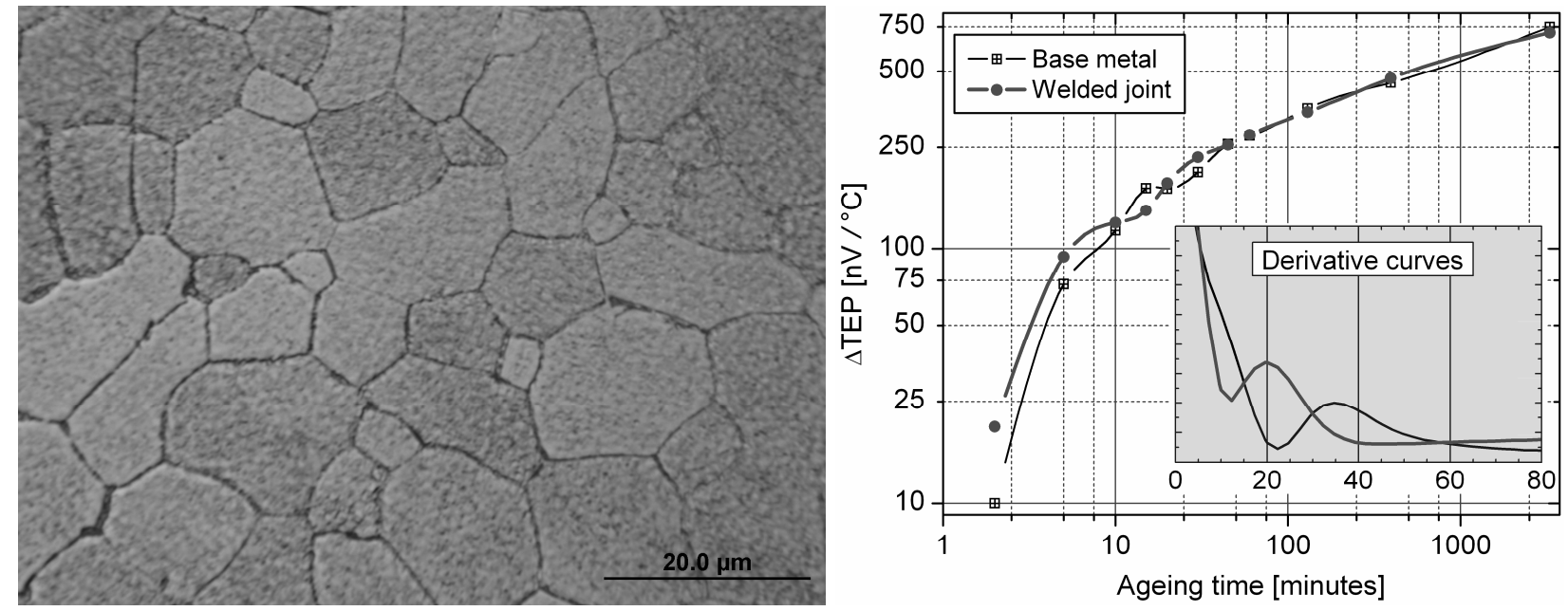

Fig. 4. Microstructure of laser weldment and the curves of TEP changing in function of ageing time 


\section{Summary}

It is well known that when duplex stainless steels are exposed to temperatures ranging from 300 to $500{ }^{\circ} \mathrm{C}$ the $\alpha$ phase formation can occur. Conventional microstructural analysis of weldments, which were thermally aged at $400{ }^{\circ} \mathrm{C}$, does not shows microscopic changes, because the precipitated phases have submicroscopic dimension. However, appearing of the $\alpha^{\prime}$ phase is sensitively detectable using thermoelectric power measurement both for cold rolled base metal and also ATIG welded and laser welded joints.

This measuring method was combined with a simple calculation that gave a useful tool for determining the incubation time of formation of the $\alpha^{\prime}$ phase. Change of TEP in function of ageing time can be transformed to derivative curves at which the first minimum shows the starting of the $\alpha$ ' phase transformation, and the next maximum signifies the end of this transformation.

The gradient of TEP changing curves only slowly approaches to zero at duplex stainless steel but much more quickly at superduplex stainless steel weldment.

In the 2205 DSS the incubation time for base material and ATIG-welded weldment is practically the same: $23-24$ minutes at $400{ }^{\circ} \mathrm{C}$. In the laser welded 2509 SDSS the incubation time of $\alpha$ phase formation is similar (22,3 min), however, in the weldment tranformation started much more quickly: after 12,2 minutes.

These results shows that thermoelectric power measurements are very powerful for characterizing phase transformations of duplex steels and welded joints.

\section{Acknowledgements}

This work was supported by the Hungarian Scientific Research Fund (T048895, K62466, K69122) and has been performed in the frame of the Bolyai János Research Grant awarded by the HAS.

\section{References}

[1] J.J. Lowke, M. Tanaka and M. Ushio, J. Phys.D: Appl. Phys., Vol. 38 (2005), p. 3438

[2] G. M. Oreper, T. W. Eagar, J. Szekely: Welding Journal, Vol. 62 (1983), p. 307

[3] N. Perry: These de Doctorat, Université De Nantes, (2000), N Ed. 82-452

[4] R. Badji: Mater. Charact., (2007) In Press

[5] S. Sire, S. Marya: C.R. Mecanique, Vol. 330 (2002), p. 83

[6] S. Sire, G. Rückert and S. Marya: Matériaux 2002, Tours, 21-25 Octobre 2002, Cm08006.pdf

[7] P.J. Modenesi, E.R. Apolinário and I.M. Pereira: J. Mater. Proc. Tech., 99 (2000), p. 260

[8] T. Paskell, C. Lundin and H. Castner: Weld. J., Vol. 76 (1997), p..57

[9] J. Niagaj: Weld. Int., Vol. 17 (2003), p. 257

[10] I. Meszaros: Phys. B-Cond. Matter, Vol. 372 (2006), p.181

[11] X. Kleber, F. Fouquet, M. Delnondedieu: Scripta Mater. Vol. 51 (2004), p. 1171

[12] V. Massardier, V. Guetaz, J. Merlin, M. Soler: Mater. Sci. Eng., A. Vol. 355 (2003), p. 299

[13] Z. Gaál, P.J. Szabó: Mater. Sci. Forum Vol. $537-538$ (2007), p. 355

[14] J. Dobranszky, P.J. Szabo, T. Berecz, V. Hrotko, M. Portko: Spectrochim. Acta, Part B. Vol. 59 (2004), p. 1781 